\title{
Clustering of Poor Dietary Habits among Adolescents Aged 12 to 15 Years in 52 Low-Income and Middle-Income Countries
}

\author{
Hui Fan $1, *\left(\mathbb{D}\right.$ and Xingyu Zhang ${ }^{2}$ (i) \\ 1 Department of Preventive Medicine, North Sichuan Medical College, Nanchong 637000, China \\ 2 Applied Biostatistics Laboratory, University of Michigan School of Nursing, Ann Arbor, MI 48109, USA; \\ zhangxyu@med.umich.edu \\ * Correspondence: nsmcfanhui@163.com
}

Received: 26 August 2020; Accepted: 15 September 2020; Published: 18 September 2020

check for updates Abstract: Very few studies have reported the co-occurrence of poor dietary habits. We thus aimed to
estimate the co-occurrence of poor dietary habits in adolescents in low-income and middle-income
countries (LMICs). Data were obtained from the Global School-Based Student Health Surveys (GSHS)
from 2009 to 2017. The suboptimal dietary factors included fast food consumption, carbonated soft
drink consumption, and low fruit and vegetable intake, which were assessed with a questionnaire
survey. We calculated the corresponding country-specific prevalence with the number of suboptimal
dietary factors. We also calculated pooled estimates across countries using a meta-analysis with
random-effects. Our study included 145,021 adolescents between 12 and 15 years of age from
52 LMICs. The prevalence of fast food consumption, carbonated soft drink consumption, and low
fruit and vegetable intake ranged from $20.9 \%$ in Pakistan to $80.0 \%$ in Thailand, from $22.4 \%$ in Kiribati
to $79.3 \%$ in Suriname, and from $45.9 \%$ in Vanuatu to $90.7 \%$ in Nepal, respectively. The prevalence of
exposure to two or three suboptimal dietary factors varied greatly across countries, ranging from
$31.8 \%$ in Pakistan to $53.8 \%$ in Nepal and from $8.6 \%$ in Vietnam to $36.4 \%$ in Suriname, respectively.
The pooled prevalence of exposure to two or three suboptimal dietary factors was $41.8 \%$ and $20.0 \%$,
respectively. Our findings indicate that poor dietary habits are frequent and tend to co-occur in
adolescents in LMICs. Country-specific policies and programs are needed to address these conditions.

Keywords: poor dietary habits; adolescents; low- and middle-income countries

\section{Introduction}

Poor dietary habits continue to be a public health crisis because they play an important role in the onset of obesity, cardiovascular disease, diabetes, cancer, and mental disorders [1-6]. They also account for one of every five deaths globally [7]. It is well established that dietary behaviors in early life track into adulthood [8], so the establishment of healthy dietary behaviors early in life is crucial in the prevention of chronic non-communicable diseases and their association with premature death [9].

Fast food consumption, carbonated soft drink consumption, and low fruit and vegetable intake in children and adolescents have received considerable attention. Global surveys have shown that these three suboptimal dietary factors are frequent in adolescents [10,11], but adolescents in high-income countries have experienced improvements in their diet quality in recent years [12,13]. In contrast, due to the high cost of a healthy diet, adolescents in low-income and middle-income countries (LMICs) may tend to reduce their intake of healthy foods and become reliant on convenience food [12]. Studies have indicated that fast food consumption, carbonated soft drink consumption, and low fruit and vegetable intake in children and adolescents tend to co-occur $[14,15]$. However, the current co-occurrence of 
these three poor dietary habits remains unclear. We therefore sought to assess this issue in adolescents in LMICs using the Global School-Based Student Health Surveys (GSHS).

\section{Materials and Methods}

\subsection{Study Population}

The GSHS is a multi-country, cross-sectional, school-based survey used to assess behavioral risk factors in adolescents $[5,6,10,11,16]$. It was initiated by the World Health Organization (WHO) and the U.S. Centers for Disease Control and Prevention. The design and methods of the GSHS have been described in detail $[5,6,10,11,16]$. In brief, in each participating country, a two-stage cluster design was used to obtain a nationally representative sample, and a self-administered questionnaire survey was conducted to collect data on health behavior. Ethical approval was obtained from the Ministry of Education or Health and an institutional review board or ethics committee in each country. The participants and their parents provided verbal or written consent. Our study used data from the GSHS 2009-2017, which were publicly available at www.who.int/chp/gshs and www.cdc.gov/gshs, and exempt under the ethical board review of the corresponding author's institution.

LMICs were based on the World Bank classification at the time of the survey for the respective countries. Public GSHS datasets were available from 75 LMICs. We excluded 23 country-specific datasets due to a lack of information regarding fast food consumption, carbonated soft drink consumption, or fruit or vegetable intake. We selected only the most recent survey if several GSHS waves were performed in a country. We also included only 12-15-year-old adolescents because data for this exact age range were available. Our study included 150,838 adolescents between 12 and 15 years of age. We then excluded 5817 adolescents with missing data for sex, food security, fast food consumption, carbonated soft drink consumption, fruit or vegetable intake. As a result, the final analysis sample consisted of 145,021 adolescents between 12 and 15 years of age from 52 LMICs (Figure S1).

\subsection{Definitions}

Sex, age, food security, fast food consumption, carbonated soft drink consumption, and low fruit and vegetable intake were assessed with a self-administered questionnaire. Adolescents who had eaten food from a fast food restaurant (country-specific examples, such as McDonalds or Burger King in Argentina) at least 1 day during the past 7 days were considered to be consumers of fast food [5]. Adolescents who had consumed carbonated soft drinks at least once per day for the past 30 days were considered to be consumers of carbonated soft drinks [10]. Low vegetable and fruit intake was defined as consumption of fewer than five servings of fruit and vegetables per day over the past 30 days $[6,16]$. The number of individual suboptimal dietary factors (fast food consumption, carbonated soft drink consumption, and low fruit and vegetable intake) were calculated.

\subsection{Statistical Analysis}

To account for the multistage sampling design, we used sampling weights to estimate the corresponding proportion and 95\% confidence intervals (CIs) for each suboptimal dietary factor in each country. In addition, to evaluate the co-occurrence of suboptimal dietary factors in each country, we calculated the corresponding proportion and $95 \% \mathrm{CI}$ with the number of suboptimal dietary factors (range, 0 to 3). We performed a meta-analysis with a random-effects model to compute the overall estimates. We also used the $\mathrm{I}^{2}$ statistic to evaluate heterogeneity among the country-specific estimates. We defined a high degree of heterogeneity with an $\mathrm{I}^{2}$ value of at least $75 \%$. We performed subgroup analyses stratified by survey year (2009-2013 vs. 2014-2017), WHO region, and food security (yes vs. no). SAS version 9.4 (SAS Institute, Cary, NC, USA) and STATA version 11.0 (Stata Corporation, College Station, TX, USA) were used to conduct the statistical analyses. 


\section{Results}

Our study included 145,021 adolescents between 12 and 15 years of age from 52 LMICs. Table 1 summarizes the characteristics of the participants across countries. The 52 LMICs were classified into five WHO regions (Americas, 14; Western Pacific, 13; Africa, 9; Eastern Mediterranean, 9; and Southeast Asia, 7). The survey year ranged from 2009 to 2017 . The overall response rate was $96.1 \%$, ranging from $87.2 \%$ in Liberia in 2017 to $99.1 \%$ in Peru in 2010. The sample sizes varied from 472 in Liberia in 2017 to 20,596 in Argentina in 2012.

Table 2 presents the prevalence of fast food consumption, carbonated soft drink consumption, and low fruit and vegetable intake. These data varied greatly across countries, ranging from $20.9 \%$ in Pakistan to $80.0 \%$ in Thailand, from $22.4 \%$ in Kiribati to $79.3 \%$ in Suriname, and from $45.9 \%$ in Vanuatu to $90.7 \%$ in Nepal, respectively. The pooled prevalence of fast food consumption, carbonated soft drink consumption, and low fruit and vegetable intake were $52.7 \%, 50.3 \%$, and $74.4 \%$, respectively.

Table 3 presents the co-occurrence of unhealthy eating habits across countries. The prevalence of exposure to two or three suboptimal dietary factors varied substantially across countries, ranging from $31.8 \%$ in Pakistan to $53.8 \%$ in Nepal and from $8.6 \%$ in Vietnam to $36.4 \%$ in Suriname, respectively. The pooled prevalence of exposure to two or three suboptimal dietary factors were $41.8 \%$ and $20.0 \%$, respectively. The incidence of exposure to two suboptimal dietary factors exceeded $41.8 \%$ in $31(59.6 \%)$ of the 52 participating countries. The incidence of exposure to all three suboptimal dietary factors exceeded $20.0 \%$ in $27(51.9 \%)$ of the 52 participating countries. Table S1 further presents the co-occurrence of unhealthy eating habits stratified by survey year (2009-2013 vs. 2014-2017), WHO region (Americas, Western Pacific, Africa, Eastern Mediterranean, and Southeast Asia), and food security (yes vs. no). The relative high incidence of exposure to two or three suboptimal dietary factors can be observed by subgroup analyses. We also noted substantial heterogeneity in the prevalence estimates of the suboptimal dietary factors (Table 3 and Table S1; all $\mathrm{I}^{2}>75 \%$ ). 
Table 1. Characteristics of all participants aged 12-15 years across countries.

\begin{tabular}{|c|c|c|c|c|c|c|}
\hline Country & Survey Year & Region & Response Rate, \% & $\mathbf{N}$ & Mean Age, Years & Boys, \% \\
\hline Afghanistan & 2014 & Eastern Mediterranean & 88.3 & 1319 & 14.1 & 53.6 \\
\hline Algeria & 2011 & Africa & 97.8 & 3408 & 13.6 & 45.8 \\
\hline Argentina & 2012 & America & 95.7 & 20,596 & 13.9 & 47.6 \\
\hline Bangladesh & 2014 & South-East Asia & 94.3 & 2597 & 14.0 & 63.8 \\
\hline Belize & 2011 & America & 97.4 & 1559 & 13.6 & 48.0 \\
\hline Benin & 2016 & Africa & 97.6 & 700 & 14.2 & 65.8 \\
\hline Bolivia & 2012 & America & 95.6 & 2682 & 14.0 & 50.1 \\
\hline Cambodia & 2013 & Western Pacific & 98.7 & 1789 & 14.1 & 48.5 \\
\hline Costa Rica & 2009 & America & 98.1 & 2223 & 14.0 & 49.8 \\
\hline Dominica & 2009 & America & 97.1 & 1272 & 13.6 & 49.9 \\
\hline Dominican Republic & 2016 & America & 94.7 & 903 & 14.3 & 48.2 \\
\hline Egypt & 2011 & Eastern Mediterranean & 94.4 & 2232 & 13.5 & 48.7 \\
\hline El Salvador & 2013 & America & 96.6 & 1560 & 14.0 & 50.6 \\
\hline Eswatini & 2013 & Africa & 97.0 & 1278 & 14.1 & 39.2 \\
\hline Fiji & 2016 & Western Pacific & 92.2 & 1417 & 14.4 & 48.9 \\
\hline Ghana & 2012 & Africa & 96.9 & 1298 & 13.9 & 48.8 \\
\hline Guatemala & 2015 & America & 94.6 & 3417 & 13.9 & 50.4 \\
\hline Guyana & 2010 & America & 95.8 & 1891 & 14.1 & 48.4 \\
\hline Honduras & 2012 & America & 95.9 & 1425 & 13.6 & 46.2 \\
\hline Indonesia & 2015 & South-East Asia & 97.5 & 8589 & 13.5 & 49.0 \\
\hline Iraq & 2012 & Eastern Mediterranean & 96.2 & 1475 & 13.9 & 54.7 \\
\hline Jamaica & 2017 & America & 95.7 & 1015 & 14.2 & 47.4 \\
\hline Kiribati & 2011 & Western Pacific & 97.2 & 1302 & 14.0 & 45.3 \\
\hline Lao People's Democratic Republic & 2015 & Western Pacific & 98.6 & 1621 & 14.5 & 47.8 \\
\hline Lebanon & 2017 & Eastern Mediterranean & 97.2 & 3254 & 13.6 & 47.1 \\
\hline Liberia & 2017 & Africa & 87.2 & 472 & 14.0 & 50.8 \\
\hline Malaysia & 2012 & Western Pacific & 99.0 & 16,106 & 14.0 & 49.5 \\
\hline Maldives & 2014 & South-East Asia & 94.1 & 1676 & 14.4 & 48.8 \\
\hline Mauritania & 2010 & Africa & 93.2 & 1197 & 14.2 & 53.4 \\
\hline
\end{tabular}


Table 1. Cont.

\begin{tabular}{|c|c|c|c|c|c|c|}
\hline Country & Survey Year & Region & Response Rate, \% & $\mathbf{N}$ & Mean Age, Years & Boys, \% \\
\hline Mongolia & 2013 & Western Pacific & 97.1 & 3599 & 13.7 & 49.4 \\
\hline Morocco & 2016 & Eastern Mediterranean & 92.4 & 3674 & 13.6 & 50.8 \\
\hline Mozambique & 2015 & Africa & 88.8 & 593 & 14.1 & 51.1 \\
\hline Namibia & 2013 & Africa & 94.9 & 1837 & 14.1 & 42.4 \\
\hline Nepal & 2015 & South-East Asia & 95.3 & 4400 & 13.7 & 47.5 \\
\hline Pakistan & 2009 & Eastern Mediterranean & 97.2 & 4860 & 14.1 & 60.7 \\
\hline Paraguay & 2017 & America & 95.4 & 1882 & 13.9 & 47.4 \\
\hline Peru & 2010 & America & 99.1 & 2338 & 14.1 & 49.8 \\
\hline Philippines & 2015 & Western Pacific & 98.8 & 6088 & 13.9 & 48.1 \\
\hline Samoa & 2011 & Western Pacific & 90.0 & 1991 & 14.0 & 47.1 \\
\hline Solomon Islands & 2011 & Western Pacific & 91.7 & 848 & 14.1 & 51.9 \\
\hline Sri Lanka & 2016 & South-East Asia & 97.3 & 2194 & 13.9 & 49.1 \\
\hline Sudan & 2012 & Eastern Mediterranean & 93.6 & 1312 & 14.2 & 51.9 \\
\hline Suriname & 2016 & America & 97.5 & 1416 & 13.8 & 46.0 \\
\hline Syrian Arab Republic & 2010 & Eastern Mediterranean & 97.1 & 2843 & 13.6 & 50.7 \\
\hline Thailand & 2015 & South-East Asia & 95.7 & 3956 & 13.7 & 49.3 \\
\hline Timor-Leste & 2015 & South-East Asia & 90.1 & 1470 & 14.1 & 46.0 \\
\hline Tonga & 2017 & Western Pacific & 97.0 & 2004 & 13.6 & 51.2 \\
\hline Tuvalu & 2013 & Western Pacific & 93.5 & 635 & 13.3 & 48.2 \\
\hline Tanzania & 2014 & Africa & 95.7 & 2502 & 13.6 & 47.0 \\
\hline Vanuatu & 2016 & Western Pacific & 95.0 & 1224 & 14.1 & 47.3 \\
\hline Vietnam & 2013 & Western Pacific & 97.4 & 1697 & 14.5 & 46.8 \\
\hline Yemen & 2014 & Eastern Mediterranean & 89.2 & 1385 & 13.8 & 56.5 \\
\hline
\end{tabular}

$\mathrm{N}=$ Sample size with complete data. 
Table 2. Prevalence of fast food consumption, carbonated soft drink consumption, and low fruit and vegetable intake.

\begin{tabular}{|c|c|c|c|}
\hline Country & $\begin{array}{c}\text { Carbonated Soft Drink Consumption, } \\
\%(95 \% \mathrm{CI})\end{array}$ & Fast Food Consumption, $\%(95 \% \mathrm{CI})$ & $\begin{array}{l}\text { Low Fruit and Vegetable Intake, } \\
\qquad \%(95 \% \mathrm{CI})\end{array}$ \\
\hline Afghanistan & $40.7(36.0,45.4)$ & $63.4(57.1,69.6)$ & $85.2(80.6,89.9)$ \\
\hline Algeria & $77.6(75.2,80.1)$ & $51.7(48.3,55.0)$ & $65.5(62.6,68.5)$ \\
\hline Argentina & $65.7(63.5,68.0)$ & $31.3(29.2,33.3)$ & $82.5(81.0,83.9)$ \\
\hline Bangladesh & $47.8(43.8,51.9)$ & $53.5(48.6,58.3)$ & $83.6(79.7,87.5)$ \\
\hline Belize & $63.8(60.4,67.1)$ & $65.7(60.8,70.6)$ & $70.5(67.8,73.3)$ \\
\hline Benin & $43.3(37.3,49.3)$ & $46.8(40.3,53.2)$ & $69.0(63.5,74.5)$ \\
\hline Bolivia & $63.3(60.8,65.7)$ & $57.0(54.5,59.6)$ & $68.4(65.7,71.1)$ \\
\hline Cambodia & $45.6(40.6,50.6)$ & $25.4(22.3,28.6)$ & $89.9(88.3,91.4)$ \\
\hline Costa Rica & $52.7(49.0,56.4)$ & $54.4(48.8,60.1)$ & $80.7(78.0,83.4)$ \\
\hline Dominica & $56.1(52.7,59.5)$ & $46.4(43.0,49.8)$ & $73.5(70.1,76.8)$ \\
\hline Dominican Republic & $74.9(73.3,76.6)$ & $46.0(40.9,51.2)$ & $81.1(77.0,85.2)$ \\
\hline Egypt & $55.2(48.8,61.6)$ & $49.0(42.5,55.5)$ & $75.2(69.6,80.8)$ \\
\hline El Salvador & $66.2(62.4,70.1)$ & $57.0(53.4,60.5)$ & $79.1(76.5,81.8)$ \\
\hline Eswatini & $45.7(42.3,49.1)$ & $41.1(37.0,45.2)$ & $79.6(75.8,83.4)$ \\
\hline Fiji & $63.3(59.1,67.5)$ & $63.8(58.4,69.2)$ & $62.7(57.1,68.4)$ \\
\hline Ghana & $53.8(46.6,61.0)$ & $69.0(60.8,77.3)$ & $65.1(60.8,69.3)$ \\
\hline Guatemala & $61.2(55.6,66.7)$ & $57.2(49.1,65.4)$ & $70.8(68.7,72.9)$ \\
\hline Guyana & $71.0(66.5,75.4)$ & $55.5(52.6,58.5)$ & $68.4(63.6,73.2)$ \\
\hline Honduras & $73.7(70.2,77.2)$ & $47.5(43.8,51.2)$ & $73.5(69.7,77.4)$ \\
\hline Indonesia & $28.9(26.5,31.4)$ & $54.7(52.1,57.2)$ & $75.3(73.3,77.3)$ \\
\hline Iraq & $53.9(50.6,57.2)$ & $55.5(50.8,60.1)$ & $73.4(69.6,77.1)$ \\
\hline Jamaica & $70.2(65.3,75.1)$ & $58.6(55.9,61.3)$ & $81.3(77.1,85.6)$ \\
\hline Kiribati & $22.4(19.5,25.4)$ & $43.7(39.4,48.0)$ & $85.4(83.4,87.4)$ \\
\hline Lao People's Democratic Republic & $58.0(50.2,65.8)$ & $44.5(38.9,50.1)$ & $81.9(79.3,84.6)$ \\
\hline Lebanon & $48.8(45.2,52.4)$ & $77.0(75.0,79.0)$ & $75.6(73.1,78.2)$ \\
\hline Liberia & $47.3(40.8,53.8)$ & $41.5(34.7,48.4)$ & $71.3(66.8,75.8)$ \\
\hline Malaysia & $31.1(29.4,32.8)$ & $48.3(46.6,49.9)$ & $69.9(68.6,71.2)$ \\
\hline Maldives & $32.5(29.4,35.6)$ & $34.6(30.5,38.6)$ & $90.2(88.5,91.8)$ \\
\hline Mauritania & $52.2(48.5,55.8)$ & $62.7(56.7,68.7)$ & $71.4(66.9,75.8)$ \\
\hline Mongolia & $33.0(30.4,35.6)$ & $55.2(49.7,60.7)$ & $78.5(76.8,80.1)$ \\
\hline Morocco & $33.4(31.4,35.4)$ & $61.8(57.9,65.7)$ & $64.1(60.3,67.9)$ \\
\hline
\end{tabular}


Table 2. Cont.

\begin{tabular}{|c|c|c|c|}
\hline Country & $\begin{array}{c}\text { Carbonated Soft Drink Consumption, } \\
\%(95 \% \mathrm{CI})\end{array}$ & Fast Food Consumption, $\%(95 \% \mathrm{CI})$ & $\begin{array}{l}\text { Low Fruit and Vegetable Intake, } \\
\qquad \%(95 \% \mathrm{CI})\end{array}$ \\
\hline Mozambique & $57.7(45.8,69.7)$ & $64.5(53.5,75.5)$ & $75.4(70.2,80.6)$ \\
\hline Namibia & $51.5(47.1,55.9)$ & $53.8(47.9,59.6)$ & $71.4(66.9,75.9)$ \\
\hline Nepal & $33.4(28.8,38.0)$ & $75.2(71.2,79.2)$ & $90.7(88.3,93.1)$ \\
\hline Pakistan & $36.5(29.7,43.3)$ & $20.9(17.5,24.3)$ & $90.0(87.9,92.1)$ \\
\hline Paraguay & $61.3(57.5,65.0)$ & $54.5(50.3,58.6)$ & $73.1(71.1,75.2)$ \\
\hline Peru & $53.2(49.5,57.0)$ & $50.1(46.0,54.1)$ & $90.2(87.4,92.9)$ \\
\hline Philippines & $37.7(34.7,40.7)$ & $51.9(46.0,57.8)$ & $74.5(72.4,76.6)$ \\
\hline Samoa & $53.5(50.3,56.6)$ & $78.8(75.3,82.3)$ & $52.1(48.2,56.0)$ \\
\hline Solomon Islands & $43.8(39.7,47.9)$ & $64.6(54.3,75.0)$ & $55.2(50.4,60.0)$ \\
\hline Sri Lanka & $26.7(23.0,30.5)$ & $42.7(36.9,48.5)$ & $75.8(72.7,79.0)$ \\
\hline Sudan & $38.7(34.2,43.2)$ & $41.2(35.1,47.3)$ & $77.1(73.7,80.5)$ \\
\hline Suriname & $79.3(76.5,82.1)$ & $63.9(60.0,67.8)$ & $71.0(68.2,73.7)$ \\
\hline Syrian Arab Republic & $30.9(27.7,34.2)$ & $42.7(37.3,48.1)$ & $84.5(82.7,86.4)$ \\
\hline Thailand & $57.0(51.5,62.6)$ & $80.0(78.0,81.9)$ & $70.0(66.7,73.2)$ \\
\hline Timor-Leste & $44.2(39.8,48.7)$ & $66.6(62.6,70.6)$ & $83.5(79.6,87.4)$ \\
\hline Tonga & $60.7(58.2,63.1)$ & $69.7(67.5,71.9)$ & $54.8(52.4,57.2)$ \\
\hline Tuvalu & $54.0(50.0,57.9)$ & $44.4(40.6,48.3)$ & $64.9(61.1,68.5)$ \\
\hline Tanzania & $47.5(42.5,52.4)$ & $35.3(30.2,40.5)$ & $65.0(60.3,69.6)$ \\
\hline Vanuatu & $41.2(37.3,45.1)$ & $57.3(52.4,62.1)$ & $45.9(41.8,49.9)$ \\
\hline Vietnam & $34.9(28.5,41.3)$ & $29.7(25.8,33.6)$ & $77.1(73.9,80.2)$ \\
\hline Yemen & $37.0(29.2,44.9)$ & $34.2(25.3,43.1)$ & $79.1(74.1,84.2)$ \\
\hline Pooled estimate & $50.3(45.6,55.0)$ & $52.7(48.4,57.0)$ & $74.4(71.8,77.0)$ \\
\hline $\mathrm{I}^{2}(\%)$ & 99.1 & 99.1 & 98.1 \\
\hline
\end{tabular}


Table 3. Clustering of unhealthy eating habits across countries.

\begin{tabular}{|c|c|c|c|c|}
\hline \multirow{2}{*}{ Country } & \multicolumn{4}{|c|}{ Number of Unhealthy Eating Habits, \% (95\% CI) } \\
\hline & 0 & 1 & 2 & 3 \\
\hline Afghanistan & $2.2(0.7,3.7)$ & $29.2(25.1,33.3)$ & $45.8(41.1,50.4)$ & $22.9(19.4,26.3)$ \\
\hline Algeria & $2.7(1.8,3.5)$ & $25.0(23.0,26.9)$ & $47.2(45.1,49.2)$ & $25.2(22.4,27.9)$ \\
\hline Argentina & $3.5(2.9,4.0)$ & $31.8(30.2,33.3)$ & $46.6(45.5,47.7)$ & $18.2(16.6,19.7)$ \\
\hline Bangladesh & $1.4(0.4,2.5)$ & $34.4(29.8,39.0)$ & $41.9(38.1,45.6)$ & $22.3(19.0,25.5)$ \\
\hline Belize & $4.0(3.0,5.0)$ & $22.0(18.5,25.5)$ & $44.0(40.2,47.8)$ & $30.0(26.8,33.2)$ \\
\hline Benin & $5.2(3.0,7.4)$ & $43.5(38.9,48.1)$ & $38.3(33.9,42.6)$ & $13.0(10.5,15.5)$ \\
\hline Bolivia & $5.2(4.3,6.1)$ & $26.8(25.4,28.2)$ & $42.2(40.6,43.7)$ & $25.9(23.9,27.8)$ \\
\hline Costa Rica & $3.8(3.0,4.6)$ & $30.0(26.4,33.6)$ & $40.7(38.1,43.2)$ & $25.5(22.4,28.7)$ \\
\hline Dominica & $5.0(3.7,6.2)$ & $33.8(31.0,36.5)$ & $41.6(38.6,44.6)$ & $19.7(17.2,22.1)$ \\
\hline Dominican Republic & $2.3(1.4,3.3)$ & $21.7(18.6,24.8)$ & $47.6(44.1,51.1)$ & $28.4(24.4,32.4)$ \\
\hline Egypt & $3.9(2.1,5.6)$ & $32.3(26.9,37.7)$ & $44.4(39.4,49.4)$ & $19.4(15.0,23.9)$ \\
\hline El Salvador & $3.6(2.6,4.7)$ & $23.1(19.4,26.7)$ & $40.5(38.4,42.6)$ & $32.7(29.1,36.3)$ \\
\hline Eswatini & $4.7(3.1,6.3)$ & $40.5(36.7,44.3)$ & $38.5(35.7,41.2)$ & $16.3(13.1,19.6)$ \\
\hline Fiji & $4.7(3.4,5.9)$ & $25.9(22.5,29.4)$ & $44.3(41.1,47.4)$ & $25.1(22.6,27.6)$ \\
\hline Ghana & $3.3(1.6,5.0)$ & $27.5(21.1,33.9)$ & $47.2(43.5,51.0)$ & $22.0(16.3,27.7)$ \\
\hline Guatemala & $5.3(4.0,6.7)$ & $25.7(22.6,28.9)$ & $43.3(40.0,46.6)$ & $25.6(21.2,30.1)$ \\
\hline Honduras & $2.5(1.7,3.3)$ & $25.8(22.3,29.3)$ & $46.2(43.7,48.6)$ & $25.5(21.9,29.1)$ \\
\hline Indonesia & $6.3(5.4,7.1)$ & $40.6(38.4,42.7)$ & $41.1(39.7,42.6)$ & $12.0(10.6,13.4)$ \\
\hline Iraq & $4.1(2.8,5.3)$ & $31.5(27.7,35.2)$ & $42.2(39.2,45.1)$ & $22.3(19.1,25.5)$ \\
\hline Jamaica & $1.7(0.8,2.6)$ & $20.1(16.4,23.8)$ & $44.5(40.7,48.3)$ & $33.7(29.9,37.5)$ \\
\hline Kiribati & $5.0(3.7,6.3)$ & $48.6(45.6,51.6)$ & $36.3(33.0,39.6)$ & $10.1(7.5,12.8)$ \\
\hline Lao People's Democratic Republic & $3.2(1.9,4.4)$ & $32.0(26.3,37.8)$ & $42.0(39.1,45.0)$ & $22.8(17.7,27.8)$ \\
\hline Lebanon & $3.2(2.5,3.9)$ & $21.3(19.3,23.3)$ & $46.6(44.6,48.6)$ & $29.0(26.6,31.4)$ \\
\hline Liberia & $5.2(3.2,7.3)$ & $42.1(36.8,47.3)$ & $40.0(36.1,43.8)$ & $12.7(9.5,15.9)$ \\
\hline
\end{tabular}


Table 3. Cont

\begin{tabular}{|c|c|c|c|c|}
\hline \multirow{2}{*}{ Country } & \multicolumn{4}{|c|}{ Number of Unhealthy Eating Habits, \% (95\% CI) } \\
\hline & 0 & 1 & 2 & 3 \\
\hline Malaysia & $10.0(9.2,10.8)$ & $42.4(41.0,43.8)$ & $36.0(34.9,37.2)$ & $11.6(10.8,12.4)$ \\
\hline Maldives & $3.4(2.2,4.6)$ & $48.5(44.7,52.3)$ & $35.6(32.4,38.8)$ & $12.5(10.3,14.7)$ \\
\hline Mauritania & $3.6(2.0,5.2)$ & $29.2(24.8,33.6)$ & $44.6(40.7,48.4)$ & $22.6(18.0,27.2)$ \\
\hline Mongolia & $5.4(4.5,6.4)$ & $37.4(33.5,41.4)$ & $42.1(39.2,45.1)$ & $15.0(12.7,17.2)$ \\
\hline Morocco & $9.1(8.0,10.2)$ & $35.0(32.6,37.4)$ & $43.3(41.3,45.4)$ & $12.5(10.5,14.5)$ \\
\hline Mozambique & $3.9(2.4,5.4)$ & $24.1(17.9,30.3)$ & $42.6(34.4,50.8)$ & $29.4(19.7,39.2)$ \\
\hline Namibia & $3.9(2.3,5.5)$ & $33.6(30.1,37.1)$ & $44.4(41.4,47.5)$ & $18.1(15.5,20.6)$ \\
\hline Pakistan & $2.8(2.1,3.5)$ & $56.2(50.5,61.9)$ & $31.8(27.6,36.0)$ & $9.2(6.8,11.6)$ \\
\hline Paraguay & $5.2(4.0,6.4)$ & $26.4(22.7,30.1)$ & $42.7(40.2,45.1)$ & $25.7(22.5,28.9)$ \\
\hline Peru & $1.5(0.9,2.1)$ & $29.9(26.9,32.9)$ & $42.1(39.9,44.3)$ & $26.5(23.8,29.1)$ \\
\hline Philippines & $7.4(6.0,8.7)$ & $36.5(32.5,40.4)$ & $40.9(37.8,44.0)$ & $15.3(12.9,17.7)$ \\
\hline Samoa & $4.5(3.3,5.7)$ & $28.0(25.4,30.5)$ & $46.1(41.9,50.4)$ & $21.4(18.6,24.2)$ \\
\hline Solomon Islands & $9.1(5.5,12.7)$ & $32.0(28.0,36.1)$ & $45.0(39.6,50.3)$ & $13.9(9.9,17.9)$ \\
\hline Sri Lanka & $9.6(7.1,12.1)$ & $46.0(41.5,50.5)$ & $33.8(29.2,38.4)$ & $10.5(8.9,12.2)$ \\
\hline Sudan & $5.7(4.0,7.5)$ & $45.3(40.9,49.7)$ & $35.1(31.6,38.6)$ & $13.8(10.9,16.8)$ \\
\hline Suriname & $2.6(1.8,3.3)$ & $17.1(14.8,19.4)$ & $43.9(40.8,46.9)$ & $36.4(33.1,39.8)$ \\
\hline Thailand & $2.7(1.9,3.5)$ & $18.4(17.0,19.8)$ & $48.0(45.5,50.6)$ & $30.8(27.5,34.2)$ \\
\hline Timor-Leste & $3.0(1.9,4.1)$ & $25.7(22.8,28.6)$ & $45.3(43.6,46.9)$ & $26.0(22.4,29.7)$ \\
\hline Tonga & $6.9(5.6,8.2)$ & $24.6(22.5,26.7)$ & $45.1(42.8,47.3)$ & $23.5(21.6,25.4)$ \\
\hline Tuvalu & $5.5(4.0,7.6)$ & $39.7(36.0,43.6)$ & $40.8(37.0,44.7)$ & $14.0(11.5,16.9)$ \\
\hline Tanzania & $9.2(7.3,11.1)$ & $44.1(40.6,47.6)$ & $36.5(33.0,40.0)$ & $10.2(8.2,12.3)$ \\
\hline Vanuatu & $12.5(10.0,14.9)$ & $40.8(37.2,44.4)$ & $36.6(33.1,40.1)$ & $10.1(7.6,12.6)$ \\
\hline Vietnam & $7.6(5.0,10.3)$ & $51.8(47.8,55.7)$ & $32.0(27.6,36.4)$ & $8.6(6.3,11.0)$ \\
\hline Yemen & $5.9(3.9,7.8)$ & $49.9(42.4,57.5)$ & $32.2(27.3,37.1)$ & $12.0(7.4,16.6)$ \\
\hline Pooled estimate & $4.6(4.0,5.2)$ & $33.3(30.8,35.9)$ & $41.8(40.7,43.0)$ & $20.0(18.1,22.0)$ \\
\hline $\mathrm{I}^{2}(\%)$ & 94.5 & 97.7 & 90.8 & 97.1 \\
\hline
\end{tabular}

The aggregated percentage within a country may not account for $100 \%$ due to the rounding error effect, which slightly affected the pooled estimate across the low-income and middle-income countries. 


\section{Discussion}

Our findings indicate that suboptimal dietary factors, especially low fruit and vegetable intake, are frequent across LMICs and that suboptimal dietary factors tend to co-occur in adolescents despite the wide variations in their prevalence across countries.

Population-based studies have confirmed the adverse health consequences of fast food consumption, carbonated soft drink consumption, and low fruit and vegetable intake [1-6]. Several hypotheses have been proposed to explain these associations. First, fast food consumption leads to increased intake of energy, fat, saturated fat, and sodium [14]. Second, carbonated soft drink consumption leads to excessive sugar intake [17]. Third, low fruit and vegetable intake result in an inadequate intake of vitamins, minerals, dietary fiber, plant sterols, and flavonoids [18]. The fast food and soft drink industries target children and adolescents as potential and main consumers [19]. As a result, a prominent epidemic of suboptimal dietary factors has been observed in adolescents [10,11].

In LMICs, a transition from traditional diets to a Western diet has been noted [20]. The GSHS and other surveys have reported a high prevalence of fast food consumption, carbonated soft drink consumption, and low fruit and vegetable intake in adolescents in most LMICs [10,11,21-24]. We used updated GSHS data and presented similar results. Observation studies have also indicated the co-occurrence of suboptimal dietary factors in children and adolescents, but such co-occurrence in adolescents in LMICs remained unclear [14,15]. Given the high cost of a healthy diet and the consequent reliance on fast food in some LMICs [12], it is necessary to clarify the aforementioned issue. Our study demonstrates that the pooled prevalence of exposure to at least two suboptimal dietary factors was $61.8 \%$ in adolescents in LMICs. Our subgroup analyses stratified by survey year, WHO region, and food security did not show significant differences. Our study also showed that the co-occurrence of suboptimal dietary factors in adolescents is prominent in most LMICs.

Our results have two key implications for the establishment of policies and programs to address suboptimal dietary factors. First, a comprehensive policy and program that includes high taxes and advertising ban for unhealthy fast food and carbonated soft drinks, a sufficient supply of inexpensive fruits and vegetables, health education, a key parental role, and behavioral interventions are needed given the co-occurrence of suboptimal dietary factors [25-29]. Second, the wide variations in the co-occurrence of suboptimal dietary factors and the substantial heterogeneity in the prevalence estimates across countries indicate that country-specific policies and programs for LMICs should be developed. The effective policies and programs are based on the social norms, cultural influences, and the context of poor dietary habits $[5,10,16]$. In addition, although the co-occurrence of suboptimal dietary factors is a universal phenomenon, we also note that a few adolescents (pooled estimate: $4.6 \%$ ) in LMICs follow the principles of healthy eating. The reasons for developing healthy eating habits are needed to clarify how to help countries establish effective policies and programs.

The strengths of our study include its nationally representative sample and comparable country-specific results due to the standard procedures used for data collection. However, our study has several limitations. First, our study includes no countries from Europe. Second, some countries may not have a sufficient sample size to estimate the co-occurrence of suboptimal dietary factors. Third, the study population was restricted to adolescents who attend school. Fourth, the suboptimal dietary factors are self-reported, which may have introduced bias. Fifth, the GSHS data for 52 LMICs were collected over a long time period (2009-2017). Direct comparisons between countries may have introduced bias due to the difference in the survey year. However, subgroup analyses stratified by survey year were performed between a narrow time interval (2009-2013 or 2014-2017), and we also observed the high incidence of exposure to two or three suboptimal dietary factors (Table S1). Of note, surveys for several countries were conducted before 2013. High-quality surveillance data about behavioral risk factors from GSHS were important in several LMICs where relevant data were scarce [16]. Consequently, we kept the country-specific data from the GSHS which were conducted before 2013. Given the transition from traditional diets to a Western diet in LMICs, the updated data for these countries were needed to assess trends over time and establish country-specific policies and 
programs. Sixth, the GSHS did not involve the clear definition of fast food. The relevant result should be interpreted with caution. Finally, we observed substantial heterogeneity in the co-occurrence of suboptimal dietary factors. Future studies should seek to explore the reasons for this heterogeneity.

\section{Conclusions}

In summary, our results suggest that poor dietary habits are frequent and tend to co-occur in adolescents in LMICs. Country-specific policies and programs are needed to address these conditions given the wide variations in their prevalence across countries.

Supplementary Materials: The following are available online at http://www.mdpi.com/1660-4601/17/18/6806/s1, Figure S1: Study flowchart; Table S1: Subgroup analyses for number of unhealthy eating habits.

Author Contributions: Conceptualization, H.F. and X.Z.; methodology, H.F. and X.Z.; software, H.F.; validation, H.F.; formal analysis, H.F.; investigation, H.F.; resources, H.F.; data curation, H.F.; writing-original draft preparation, H.F.; writing - review and editing, H.F. and X.Z.; supervision, H.F. and X.Z.; project administration, H.F.; funding acquisition, H.F. All authors have read and agreed to the published version of the manuscript.

Funding: This research was funded by the PhD Funding Program of North Sichuan Medical College (CBY18-QD02), the Key Subject Development Program of North Sichuan Medical College (NSMC-M-18-19), and Funding Program of Primary Health Development Research Center of Sichuan Province (SWFZ20-Q-045).

Acknowledgments: We thank the WHO and US Centers for Disease Control for sharing the GSHS datasets.

Conflicts of Interest: The authors declare no conflict of interest.

\section{References}

1. Aune, D.; Giovannucci, E.; Boffetta, P.; Fadnes, L.T.; Keum, N.; Norat, T.; Greenwood, D.C.; Riboli, E.; Vatten, L.J.; Tonstad, S. Fruit and vegetable intake and the risk of cardiovascular disease, total cancer and all-cause mortality-A systematic review and dose-response meta-analysis of prospective studies. Int. J. Epidemiol. 2017, 46, 1029-1056. [CrossRef] [PubMed]

2. Ley, S.H.; Hamdy, O.; Mohan, V.; Hu, F.B. Prevention and management of type 2 diabetes: Dietary components and nutritional strategies. Lancet 2014, 383, 1999-2007. [CrossRef]

3. Chazelas, E.; Srour, B.; Desmetz, E.; Kesse-Guyot, E.; Julia, C.; Deschamps, V.; Druesne-Pecollo, N.; Galan, P.; Hercberg, S.; Latino-Martel, P.; et al. Sugary drink consumption and risk of cancer: Results from NutriNet-Santé prospective cohort. BMJ 2019, 366, 12408. [CrossRef] [PubMed]

4. TeMorenga, L.; Mallard, S.; Mann, J. Dietary sugars and body weight: Systematic review and meta-analyses of randomised controlled trials and cohort studies. BMJ 2012, 346, e7492. [CrossRef] [PubMed]

5. Jacob, L.; Stubbs, B.; Firth, J.; Smith, L.; Haro, J.M.; Koyanagi, A. Fast food consumption and suicide attempts among adolescents aged 12-15 years from 32 countries. J. Affect. Disord. 2020, 266, 63-70. [CrossRef] [PubMed]

6. Liu, M.W.; Chen, Q.T.; Jr Towne, S.D.; Zhang, J.; Yu, H.J.; Tang, R.; Gasevic, D.; Wang, P.G.; He, Q.Q. Fruit and vegetable intake in relation to depressive and anxiety symptoms among adolescents in 25 low- and middle-income countries. J. Affect. Disord. 2020, 261, 172-180. [CrossRef]

7. GBD 2017 Diet Collaborators. Health effects of dietary risks in 195 countries, 1990-2017: A systematic analysis for the Global Burden of Disease Study 2017. Lancet 2019, 393, 1958-1972. [CrossRef]

8. Lien, N.; Lytle, L.A.; Klepp, K.I. Stability in consumption of fruit, vegetables, and sugary foods in a cohort from age 14 to age 21. Prev. Med. 2001, 33, 217-226. [CrossRef]

9. Akseer, N.; Al-Gashm, S.; Mehta, S.; Mokdad, A.; Bhutta, Z.A. Global and regional trends in the nutritional status of young people: A critical and neglected age group. Ann. N. Y. Acad. Sci. 2017, 1393, 3-20. [CrossRef]

10. Yang, L.; Bovet, P.; Liu, Y.; Zhao, M.; Ma, C.; Liang, Y.; Xi, B. Consumption of Carbonated Soft Drinks among Young Adolescents Aged 12 to 15 Years in 53 Low- and Middle-Income Countries. Am. J. Public Health 2017, 107, 1095-1100. [CrossRef]

11. Beal, T.; Morris, S.S.; Tumilowicz, A. Global Patterns of Adolescent Fruit, Vegetable, Carbonated Soft Drink, and Fast-Food Consumption: A Meta-Analysis of Global School-Based Student Health Surveys. Food Nutr. Bull. 2019, 40, 444-459. [CrossRef] [PubMed] 
12. Vereecken, C.; Pedersen, T.P.; Ojala, K.; Krølner, R.; Dzielska, A.; Ahluwalia, N.; Giacchi, M.; Kelly, C. Fruit and vegetable consumption trends among adolescents from 2002 to 2010 in 33 countries. Eur. J. Public Health 2015, 25, 16-19. [CrossRef] [PubMed]

13. Shan, Z.; Rehm, C.D.; Rogers, G.; Ruan, M.; Wang, D.D.; Hu, F.B.; Mozaffarian, D.; Zhang, F.F.; Bhupathiraju, S.N. Trends in Dietary Carbohydrate, Protein, and Fat Intake and Diet Quality among US Adults, 1999-2016. JAMA 2019, 322, 1178-1187. [CrossRef] [PubMed]

14. Paeratakul, S.; Ferdinand, D.P.; Champagne, C.M.; Ryan, D.H.; Bray, G.A. Fast-food consumption among US adults and children: Dietary and nutrient intake profile. J. Am. Diet. Assoc. 2003, 103, 1332-1338. [CrossRef]

15. Cullen, K.W.; Ash, D.M.; Warneke, C.; de Moor, C. Intake of soft drinks, fruit-flavored beverages, and fruits and vegetables by children in grades 4 through 6. Am. J. Public Health 2002, 92, 1475-1478. [CrossRef]

16. Caleyachetty, R.; Echouffo-Tcheugui, J.B.; Tait, C.A.; Schilsky, S.; Forrester, T.; Kengne, A.P. Prevalence of behavioural risk factors for cardiovascular disease in adolescents in low-income and middle-income countries: An individual participant data meta-analysis. Lancet Diabetes Endocrinol. 2015, 3, 535-544. [CrossRef]

17. Reedy, J.; Krebs-Smith, S.M. Dietary sources of energy, solid fats, and added sugars among children and adolescents in the United States. J. Am. Diet. Assoc. 2010, 110, 1477-1484. [CrossRef]

18. Slavin, J.L.; Lloyd, B. Health benefits of fruits and vegetables. Adv. Nutr. 2012, 3, 506-516. [CrossRef]

19. Powell, L.M.; Harris, J.L.; Fox, T. Food marketing expenditures aimed at youth: Putting the numbers in context. Am. J. Prev. Med. 2013, 45, 453-461. [CrossRef]

20. Popkin, B.M.; Adair, L.S.; Ng, S.W. Global nutrition transition and the pandemic of obesity in developing countries. Nutr. Rev. 2012, 70, 3-21. [CrossRef]

21. Darfour-Oduro, S.A.; Buchner, D.M.; Andrade, J.E.; Grigsby-Toussaint, D.S. A comparative study of fruit and vegetable consumption and physical activity among adolescents in 49 Low-and-Middle-Income Countries. Sci. Rep. 2018, 8, 1623. [CrossRef] [PubMed]

22. Ratnayake, N.; Ekanayake, L. Soft drink consumption in Sri Lankan adolescents. Public Health Nutr. 2012, 15, 1333-1337. [CrossRef] [PubMed]

23. Stern, D.; Piernas, C.; Barquera, S.; Rivera, J.A.; Popkin, B.M. Caloric beverages were major sources of energy among children and adults in Mexico, 1999-2012. J. Nutr. 2014, 144, 949-956. [CrossRef] [PubMed]

24. Zhao, Y.; Wang, L.; Xue, H.; Wang, H.; Wang, Y. Fast food consumption and its associations with obesity and hypertension among children: Results from the baseline data of the Childhood Obesity Study in China Mega-cities. BMC Public Health 2017, 17, 933. [CrossRef] [PubMed]

25. Popkin, B.M.; Hawkes, C. Sweetening of the global diet, particularly beverages: Patterns, trends, and policy responses. Lancet Diabetes Endocrinol. 2016, 4, 174-186. [CrossRef]

26. Thomson, C.A.; Ravia, J. A systematic review of behavioral interventions to promote intake of fruit and vegetables. J. Am. Diet. Assoc. 2011, 111, 1523-1535. [CrossRef]

27. Hirvonen, K.; Bai, Y.; Headey, D.; Masters, W.A. Affordability of the EAT-Lancet reference diet: A global analysis. Lancet Glob. Health 2020, 8, 59-66. [CrossRef]

28. Pem, D.; Jeewon, R. Fruit and Vegetable Intake: Benefits and Progress of Nutrition Education Interventions-Narrative Review Article. Iran. J. Public Health 2015, 44, 1309-1321.

29. Di Noia, J.; Byrd-Bredbenner, C. Determinants of fruit and vegetable intake in low-income children and adolescents. Nutr. Rev. 2014, 72, 575-590. [CrossRef]

(C) 2020 by the authors. Licensee MDPI, Basel, Switzerland. This article is an open access article distributed under the terms and conditions of the Creative Commons Attribution (CC BY) license (http://creativecommons.org/licenses/by/4.0/). 